\title{
Diagnostic Reference Levels of Adults CT-Scan Imaging in Cameroon: A Pilot Study of Four Commonest CT-Protocols in Five Radiology Departments
}

\author{
Boniface Moifo ${ }^{1,2}$, Jean Roger Moulion Tapouh1,3, Mathurin Neossi Guena ${ }^{4}$, \\ Thierry Ndzana Ndah', Richard Ndi Samba5, Augustin Simo5
}

\author{
${ }^{1}$ Department of Radiology and Radiation Oncology, Faculty of Medicine and Biomedical Sciences, The University of Yaoundé 1, \\ Yaounde, Cameroon \\ ${ }^{2}$ Radiology Department, Yaounde Gynaeco-Obstetric and Pediatric Hospital, Yaounde, Cameroon \\ ${ }^{3}$ Radiology Department, Yaounde University Teaching Hospital, Yaounde, Cameroon \\ ${ }^{4}$ Department of Biomedical Sciences, University of Ngaoundere, Ngaoundere, Cameroon \\ ${ }^{5}$ National Radiation Protection Agency (ANRP), Yaounde, Cameroon \\ Email: `bmoifo@yahoo.fr, tapouh@yahoo.fr, mneossiguena@yahoo.fr, ndzanathierry@yahoo.fr, samba_ndi@yahoo.co.uk, \\ augsimo@yahoo.fr
}

How to cite this paper: Moifo, B., Moulion Tapouh, J.R., Neossi Guena, M., Ndzana Ndah, T., Samba, R.N. and Simo, A. (2017) Diagnostic Reference Levels of Adults CT-Scan Imaging in Cameroon: A Pilot Study of Four Commonest CT-Protocols in Five Radiology Departments. Open Journal of Medical Imaging, 7, 1-8. https://doi.org/10.4236/ojmi.2017.71001

Received: November 11, 2016

Accepted: January 22, 2017

Published: January 25, 2017

Copyright $\odot 2017$ by authors and Scientific Research Publishing Inc. This work is licensed under the Creative Commons Attribution International License (CC BY 4.0).

http://creativecommons.org/licenses/by/4.0/ (c) (i) Open Access

\begin{abstract}
CT-scan is the most irradiating tool in diagnostic radiology. For 5\% - 10\% of diagnostic X-ray procedures, it is responsible for $34 \%$ of irradiation according to UNSCEAR. Patients radiation protection must therefore be increased during CT-scan procedures. This requires the rigorous application of optimization principle which imposes to have "diagnostic reference levels". Objective: The aim of this study was to determine the diagnostic reference levels (DRLs) of the four most frequent CT-scans examinations of adults in Cameroon. Material and Method: It was a cross-sectional pilot study carried out from April to September 2015 in five health facilities using CT-scan in Cameroon. The studied variables were: patients age and sex, type of CT-scan examination (cerebral, chest, abdomino-pelvic, lumbar spine), Used of IV contrast (IV-/ $\mathrm{IV}+)$, acquisition length, time of tube rotation, voltage $(\mathrm{kV}), \mathrm{mAs}$, pitch, thickness of slices, CTDIvol and DLP. For each type of examination, at least 30 patients were included per center, consecutively on the randomly predetermined days. The DRL for each type of examination was defined as the 75th percentile of its PDL and CTDIvol. Results: Of the 696 examinations, $41.2 \%$ were cerebral, $26.9 \%$ abdomino-pelvic, $17.7 \%$ lumbar spine and $14.2 \%$ chest. The mean age of patients was $52 \pm 15$ years [ 20 - 90 years], $58.9 \%$ were 50 years and older. The sex-ratio was 1.26 (55.9\% males). The CT machines were 4,8 and 16 multidetectors. The 75th percentile of DLP or DRLs [standard deviation] was:
\end{abstract}


$[1150 \pm 278 \mathrm{mGy} \cdot \mathrm{cm}],[770 \pm 477 \mathrm{mGy} \cdot \mathrm{cm}],[720 \pm 170 \mathrm{mGy} \cdot \mathrm{cm}]$ and $[715 \pm$ $187 \mathrm{mGy} \cdot \mathrm{cm}]$ respectively for cerebral, lumbar spine, abdominopelvic and chest CT-scans. Taking in consideration the number of detectors, the 75 th percentile of the Dose-Length product decreased with the increase number of detectors for cerebral examinations but was the highest with 16 MDCT for the abdominopelvic, lumbar spine and chest CT-scans. For the chest and lumbar spine examinations, there was a significant increase in patient-dose with the increase in the number of detectors. Conclusion: Our DRLs values lie between the norms of some European countries and those of some African countries. There is remarquable variation in dose for the commonest CTscans examinations in Cameroon, requiring then an optimization process from these determined DRLs and establishment of national DRLs. Special attention to optimization should be paid when using 16 MDCT.

\section{Keywords}

Optimization, Diagnostic Reference Levels, Dose Length Product, CT Dose Index, Patient Dose, Radiation Protection, Best Practices

\section{Introduction}

Computed tomography (CT-scan) is known as the most important medical cause of human exposure due to ionizing radiations in diagnostic radiology. In its 2008 annual report, the UN Scientific Committee (UNSCEAR) estimated that, on a global scale, CT-scans accounted for only $5 \%$ of medical imaging examinations (versus $27 \%$ for chest X-rays) but contributed $34 \%$ to the annual collective dose (3\% for chest $\mathrm{x}$-ray) [1]. Patients radiation protection must therefore be increased during CT-scan procedures.

With rapid and constant technological developments, CT-scan has become the most requested examination in numerous clinical situations. Some indications, such as pretherapethic workup of various malignant tumors and total bodyscan, require the exploration of several regions of the body, and thus the increase of patient-dose [2]. The use of CT in pediatric diseases is also increasing, especially in resource-limited countries where MRI is scarce. Sub-Saharan Africa countries as Cameroon experienced the increase number of CT-scan machines in public and private health facilities. In 2016 more than 20 CT machines are available in Cameroon compare to five in 2004.

To reduce patients' exposition to $\mathrm{x}$-rays, the principles of justification and optimization are the cornerstones [3]. Systematic use of clinical imaging referral guidelines is the key for justification [4]. The principle of optimization is the responsibility of manufacturers (development of less and less irradiating technologies guaranteeing good image quality), the radiographers and radiologists who must endeavor to use the lowest doses for adequate image quality (ALARA). The reduction of patient-dose requires then the rigorous application of optimization principle which imposes to have "diagnostic reference levels" (DRLs) as an 
optimization tool. They are a simple benchmark for identifying situations where the patient's dose levels are abnormally high. Any user of ionizing radiation should refer to these DRLs to evaluate his practice [5]. In CT examinations, the CT dose index (CTDI), the Dose Length Product (DLP) and the effective dose are the most common used dosimetric quantities for DRLs [2] [6].

97/43 Euratom Directive imposes to each state member to develop and use DRLs. In Cameroon, despite the increasing number of CT-machines, the Diagnostic Reference Levels have not yet been established. Previous studies have shown great variability in the dose delivered to patients for the same types of examinations.

This pilot study was to determine the diagnostic reference levels (DRLs) of the four commonest CT-scans examinations of adults in Cameroon, to provide initial quantitative benchmarks to enable radiology professionals to optimize their Practice and to enrich the continental and international databases on patient-doses.

\section{Materials and Methods}

\section{Study Design and Period}

From April to September 2015 (06 months), we carried out a descriptive cross-sectional study in medical imaging services of five health facilities in 03 regions of Cameroon (Center, Littoral and West). $60 \%$ to $70 \%$ of CT-scan machines are concentrated in Yaounde (Center) and Douala (Littoral).

\section{Sample}

Eight potential services with a CT-scan was contacted, five participated to this pilot study, three were not included (2 non-functional machines during the study period and 1 refusal to participate). In each of the 05 services included, we randomly selected at least 30 acquisitions (minimum require is 20 acquisitions) for each of the four commonest CT examinations: cerebral, chest, abdominopelvic and lumbar spine. All acquisitions were helical mode.

\section{Variables}

The dosimetric quantities recorded were the Dose Length Product (DLP) in mGy.cm (milliGray.centimeter) and the Volumetric CT Dose Index (CTDIvol) in mGy (milliGray). Other studied variables were: patients age and sex, type of CT-scan examination (cerebral, chest, abdomino-pelvic, lumbar spine), Used of IV contrast (IV-/IV+), acquisition length, time of tube rotation, voltage $(\mathrm{kV})$, $\mathrm{mAs}$, pitch, and thickness of slices.

\section{Statistical Analysis}

The data were collected and analyzed using SPSS software version 2.0. The recommended Diagnostic Reference Levels for each protocol was the 75th percentile of the doses for this examination in the entire sample.

\section{Ethical Considerations}

We requested and obtained the ethical clearance of the Institutional Ethics Committee of the Faculty of Medicine and Biomedical Sciences (Yaounde) and the recruitment authorizations of the head of the service in which the study was 
conducted.

\section{Results}

Of the 696 CT-examinations recorded, $41.2 \%$ were cerebral, $26.9 \%$ abdominopelvic, $17.7 \%$ lumbar spine and $14.2 \%$ chest. The mean age of patients was $52 \pm$ 15 years [ 20 - 90 years] with $58.9 \%$ aged 50 -year-old and more and $55.9 \%$ males (sex-ratio 1.26). The CT machines were 4,8 and 16 multidetectors (GE, Hitachi, Shimazu)

\section{Acquisition Parameters}

In the five services, all protocols were helical with a voltage of $120 \mathrm{kV}$. The maximum mAs was recorded for lumbar CT-scans (213 mAs average) with highly variable acquisition parameters (Table 1).

\section{Patient-Doses for Four Commonest CT-Exams}

The highest DLP was observed on cerebral CT-scans (mean $944 \mathrm{mGy} \cdot \mathrm{cm}$ ) but the greatest variability of patient-doses was found in the lumbar spine CT with differences up to 16 times (Table 2).

$75^{\text {th }}$ Percentiles of CTDIvol and DLP Compared to Some Countries' Values

The 75th percentile of the distribution of DLPs in our sample allowed us to determine DRLs for cerebral, chest, abdominopelvic and lumbar spine CT-scans in adults in Cameroon (Table 3 and Figure 1).

$75^{\text {th }}$ Percentiles of DLP According to the Number of Detectors of CT Machines

Taking in consideration the number of detectors, the 75th percentile of the Dose-Length product decreased with the increase number of detectors for cerebral examinations but was the highest with 16 MDCT for the abdominopelvic, lumbar spine and chest CT-scans (Table 4). For the chest and lumbar spine examinations,

Table 1. Acquisition parameters for the four commonest CT-exams : mean [range].

\begin{tabular}{cccc}
\hline CT-exam types & $\mathrm{kV}$ & $\mathrm{mAs}$ & Lenght of acquisition $(\mathrm{cm})$ \\
\hline Cerebral & 120 & $204[120-384]$ & $20[10-47]$ \\
Chest & 120 & $183[90-267]$ & $36[26-57]$ \\
Abdominopelvic & 120 & $160[24-246]$ & $44[26-65]$ \\
Lombar spine & 120 & $213[38-380]$ & $30[12-54]$
\end{tabular}

Table 2. Means and ranges of CTDIvol and DLP for each group of CT-scans procedures.

\begin{tabular}{ccccc}
\hline CT protocols & \multicolumn{2}{c}{ CTDIvol $(\mathrm{mGy})$} & \multicolumn{2}{c}{ DLP (mGy·cm) } \\
\hline Mean & Range & Mean & Range \\
Cerebral & 47.7 & $10-82$ & 944 & $327-1849$ \\
Chest & 14.84 & $6-26$ & 531 & $212-919$ \\
Abdominopelvic & 13.7 & $7-51$ & 620 & $304-971$ \\
Lombar spine & 26.13 & $7-62$ & 793 & $136-2298$ \\
\hline
\end{tabular}


Table 3. DRLs in CT imaging from our study compared with other studies.

\begin{tabular}{ccccccccc}
\hline & \multicolumn{2}{c}{ Our study DRLs } & \multicolumn{2}{c}{ France 2011 [7] } & \multicolumn{2}{c}{ Kenya 2015 [8] } & \multicolumn{2}{c}{ Iran 2015 [6] } \\
\hline CT protocols & CTDI & DLP & CTDI & DLP & CTDI & DLP & CTDI & DLP \\
\hline Cerebral & $\mathbf{5 2}$ & $\mathbf{1 1 5 1}$ & 65 & 1050 & 61 & 1612 & 43 & 700 \\
Chest & $\mathbf{2 2}$ & $\mathbf{7 1 5}$ & 15 & 475 & 19 & 895 & 10 & 330 \\
Abdominopelvic & $\mathbf{1 5}$ & $\mathbf{7 1 6}$ & 17 & 800 & $/$ & $/$ & 10 & 750 \\
Lombar spine & $\mathbf{2 5}$ & $\mathbf{7 6 9}$ & 45 & 700 & 20 & 712 & $/$ \\
\hline
\end{tabular}

${ }^{*} \mathrm{CTDI}(\mathrm{mGy}) ;{ }^{* *} \mathrm{DLP}(\mathrm{mGy} \cdot \mathrm{cm})$.
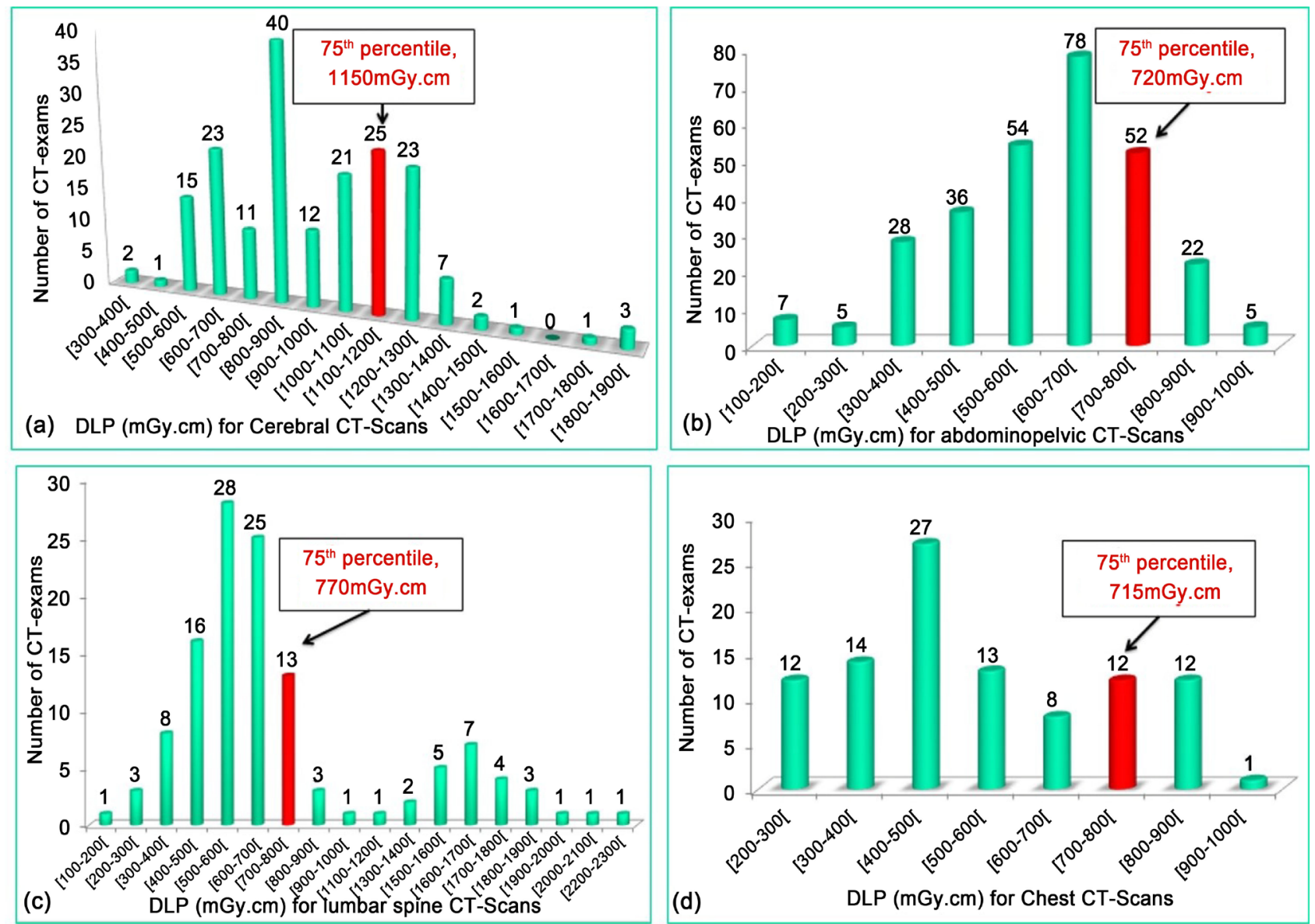

Figure 1. DRLs in ours ample : $1150 \mathrm{mGy} \cdot \mathrm{cm}$ for cerebral CT (a), $720 \mathrm{mGy} \cdot \mathrm{cm}$ for abdominopelvic CT (b), $770 \mathrm{mGy} \cdot \mathrm{cm}$ for lumbar spine CT (c) and $715 \mathrm{mGy} \cdot \mathrm{cm}$ for Chest CT (d).

Table 4. DRLs according to the number of detectors of CT-machine.

\begin{tabular}{cccc}
\hline $7^{\text {th }}$ percentile of DLP $(\mathrm{mGy} \cdot \mathrm{cm})$ & $\mathbf{4}$ MDCT & $\mathbf{8}$ MDCT & 16 MDCT \\
\hline DRLs for cerebral CT & 1300 & 1146 & 662 \\
DRLs for abdominopelvic CT & 725 & 668 & 796 \\
DRLs for lumbar spine CT & 705 & 622 & 1728 \\
DRLs for chest CT & 505 & 512 & 812 \\
\hline
\end{tabular}

MDCT $=$ mutidetectors CT. 
there was a significant increase in patient-dose with the increase in the number of detectors.

\section{Discussion}

We carried out this descriptive study in order to propose DRLs in Cameroon for four commonest adults CT-scans examinations. The $75^{\text {th }}$ percentile of DLP for cerebral, chest, abdominopelvic and lumbar spine CT-scans were respectively $1150 \mathrm{mGy} \cdot \mathrm{cm}, 715 \mathrm{mGy} \cdot \mathrm{cm}, 716 \mathrm{mGy} \cdot \mathrm{cm}$ and $770 \mathrm{mGy} \cdot \mathrm{cm}$.

The DRLs from our study are globally higher than those in France (2011), apart from the abdominopelvic protocol. On the other hand, our DRLs are lower than those in Kenya (2015) for cerebral and chest CT-scans. Our patient-doses are then between DRLs of an industrialized country as France with very modern facilities and advance culture of optimization, and the DRLs of one country like Kenya which is comparable to Cameroon. While waiting for a national campaign for establishing DRLs, those determined in this sdudy can be used for optimization. Radiologist physicians should systematic include notification of DLP on radiological reports [5].

Higher DRLs are observed on cerebral CT-scans as in Iran, Kenya and France [6] [7] [8]; this can be explained by the presence of numerous high attenuating bony structures in the skull requiring the application of high doses for adequate images quality.

The great variability observed in patient-doses, mainly for the lumbar spine, is reflecting differences in protocols used in different services and the absence of DRLs on which professional could refer to self-regulate their practice. Several actions are to be undertaken to limit the irradiation of patients: lowering the high voltage $(\mathrm{kV})$ and the tube current $(\mathrm{mAs})$, reducing the scanning length and the tube rotation time, increasing slice thickness or pitch, using auto-exposure control or dose reduction software, and the training of staff [2] [6]. Regular awareness of medical imaging professional on radiation protection during staff, meetings and national and international meetings/workshops would also disseminate best practice and harmonize the best CT-scan protocols. There is also need for quality control of CT Equipment and audit of CT practices to alert professional in case of inappropriate practice.

This study was confronted with some limitations. First, the size of the sample, limited to five services due to a lack of resources to cover the whole country, some non functional machines during the data collection and the opposition of some health facilities. The second limitation is the absence of equipment to carry out a quality control on the machines before data collection. These limits are characteristic of a resource-limited country like ours and reflect the reality of everyday practice.

Despite these limitations, we have produced preliminary results which can already be used for optimization while waiting for national study for establishment of DRLs with the assistance of the competent authorities. Further work is also required to establish DRLs in pediatric CT and conventional radiography in 
Cameroon.

\section{Conclusion}

In Cameroon, DLP values of $1150 \mathrm{mGy} \cdot \mathrm{cm}, 770 \mathrm{mGy} \cdot \mathrm{cm}, 720$ and $715 \mathrm{mGy} \cdot \mathrm{cm}$ respectively for cerebral, lumbar spine, abdominopelvic and chest CT-scans can be used as diagnostic reference levels in CT imaging in adults. These values lie between the norms of European countries and those of African countries. There is remarquable variation in dose on the most common CT-scans examinations in Cameroon, requiring then an optimization process from these determined DRLs and establishment of national DRLs. Special attention to optimization should be paid when using 16 MDCT.

\section{Acknowledgements}

For the staff members of Yaounde General Hospital, Centre Medical la Cathedrale, Centre Medico-social d'Essos, Douala General Hospital and Bafoussam Regional Hospital. Special thanks for IAEA and ANRP for workshops in radiation protection through Project RAF9044/9002/01 (Establishment and Utilisation of DRLs) and RAF/9/053/9012/03 (Optimization in Pediatric Imaging)

\section{Declaration of Interest}

The authors declare to have no competing interest in relation to this article.

\section{References}

[1] United Nations (Ed) (2010) Sources and Effects of Ionizing Radiation: United Nations Scientific Committee on the Effects of Atomic Radiation: UNSCEAR 2008 Report to the General Assembly, with Scientific Annexes. United Nations, New York.

[2] Dougeni, E., Faulkner, K. and Panayiotakis, G. (2012) A Review of Patient Dose and Optimisation Methods in Adult and Paediatric CT Scanning. European Journal of Radiology, 81, e665-e683. https://doi.org/10.1016/j.ejrad.2011.05.025

[3] Ducou le Pointe, H. (2013) Computed tomography and radioprotection: Knowing and acting. Diagn Interv Imaging, 94, 361-363. https://doi.org/10.1016/j.diii.2013.03.015

[4] Société Française de Radiologie (2013) Guide Du Bon Usage Des Examens D’imagerie Médicale. Editions SFR, Paris. http://gbu.radiologie.fr/

[5] Cordoliani, Y.-S. (2010) Les enjeux de la radioprotection en imagerie médicale: The Issues of Radiation Protection in Medical Imaging. Journal de Radiologie, 91, 11841185. https://doi.org/10.1016/S0221-0363(10)70170-0

[6] Najafi, M., Deevband, M.R., Ahmadi, M. and Kardan, M.R. (2015) Establishment of Diagnostic Reference Levels for Common Multi-Detector Computed Tomography Examinations in Iran. Australasian Physical and Engineering Science in Medicine, 38, 603-609.

[7] Arrêté du 24 Octobre 2011 relatif aux niveaux de référence diagnostiques en radiologie et en médecine nucléaire. Journal Officiel de la République Française du 14 janvier 2012, Texte 22 sur 147., NOR: ETSP1129093A.

[8] Korir, G.K., Wambani, J.S., Korir, I.K., Tries, M.A. and Boen, P.K. (2015) National 
Diagnostic Reference Level Initiative for Computed Tomography Examinations in Kenya. Radiation Protection Dosimetry, 168, 242-252.

https://doi.org/10.1093/rpd/ncv020

\section{Abreviations Note}

CT: Computed tomography;

CTDI: CT dose index;

DLP: Dose Length Product;

MDCT: Multidetector CT;

mGy: milliGray;

$\mathrm{mGy} \cdot \mathrm{cm}$ : milliGray·centimeter.

Submit or recommend next manuscript to SCIRP and we will provide best service for you:

Accepting pre-submission inquiries through Email, Facebook, LinkedIn, Twitter, etc. A wide selection of journals (inclusive of 9 subjects, more than 200 journals)

Providing 24-hour high-quality service

User-friendly online submission system

Fair and swift peer-review system

Efficient typesetting and proofreading procedure

Display of the result of downloads and visits, as well as the number of cited articles

Maximum dissemination of your research work

Submit your manuscript at: http://papersubmission.scirp.org/

Or contact ojmi@scirp.org 